\title{
Heparan sulfate proteoglycan triggers focal adhesion kinase signaling during Trypanosoma cruzi invasion
}

\author{
Tatiana G Melo, Eveline A Coutinho, Mirian Claudia S Pereira/ ${ }^{+}$ \\ Fundação Oswaldo Cruz-Fiocruz, Instituto Oswaldo Cruz, Laboratório de Ultraestrutura Celular, Rio de Janeiro, RJ, Brasil
}

BACKGROUND Trypanosoma cruzi, the etiologic agent of Chagas disease, is capable of triggering different signaling pathways that modulate its internalisation in mammalian cells. Focal adhesion kinase (FAK), a non-receptor tyrosine kinase protein, has been demonstrated as a mechanism of T. cruzi invasion in cardiomyocytes. Since the involved cell surface receptors are not yet known, we evaluated whether heparan sulfate proteoglycans (HSPG), a molecule involved in T. cruzi recognition and in the regulation of multiple signaling pathways, are able to trigger the FAK signaling pathway during T. cruzi invasion.

METHODS To investigate the role of HSPG in the regulation of the FAK signaling pathway during trypomastigote entry, we performed heparan sulfate (HS) depletion from the cardiomyocyte surface by treatment with heparinase I or $p$-nitrophenyl$\beta$-D-xylopyranoside ( $p$-n-xyloside), which abolishes glycosaminoglycan (GAG) attachment to the proteoglycan core protein. Wild-type (CHO-k1) and GAG-deficient Chinese hamster ovary cells (CHO-745) were also used as an approach to evaluate the participation of the HSPG-FAK signaling pathway. FAK activation (FAK Tyr ${ }^{397}$ ) and spatial distribution were analysed by immunoblotting and indirect immunofluorescence, respectively.

FINDINGS HS depletion from the cardiomyocyte surface inhibited FAK activation by $T$. cruzi. Cardiomyocyte treatment with heparinase I or $p$-n-xyloside resulted in $34 \%$ and $28 \%$ FAK phosphorylation level decreases, respectively. The experiments with the CHO cells corroborated the role of HSPG as a FAK activation mediator. T. cruzi infection did not stimulate FAK phosphorylation in CHO-745 cells, leading to a 36\% reduction in parasite invasion. FAK inhibition due to the PF573228 treatment also impaired T. cruzi entry in CHO-k1 cells.

MAIN CONCLUSION Jointly, our data demonstrate that HSPG is a key molecule in the FAK signaling pathway activation, regulating $T$. cruzi entry.

Key words: heparan sulfate proteoglycans - focal adhesion kinase - Trypanosoma cruzi - signaling pathway

Chagas disease, a neglected tropical disease caused by Trypanosoma cruzi, is widespread worldwide, due to migration flows from Latin America to other continents ${ }^{(1)}$ and is a leading cause of severe cardiomyopathy. (2) This disease affects 8 million people, mainly in Latin America, and is responsible for 677,000 years of life lost due to disability or death. ${ }^{(3)}$ T. cruzi transmission occurs through vector and non-vector-borne modes (congenital, iatrogenic, transplantation and oral routes), alerting to the need to implement public policies to control this silent disease in non-endemic countries. T. cruzi dissemination depends on host colonisation, escape from host defenses, replication and parasite persistence in the vertebrate host. (4) Mammalian cell entry is a requirement for T. cruzi proliferation and spread within the vertebrate host. A repertoire of molecules on the surface of mammalian cells, including galectin-3, cytokeratin 18 , fibronectin, laminin, heparan sulfate proteoglycan (HSPG), integrin, LDL and bradykinin receptors, modulates $T$. cruzi recognition and

doi: 10.1590/0074-02760200143

Financial support: FIOCRUZ (Programa Estratégico de Apoio à Pesquisa em

Saúde - Papes VI/CNPq - Grant 421856/2017-3 and 424015/2018-8 to MCSP); FAPERJ (Grant E-26/010.001548/2014 and E-26/110.553/2014 to MCSP).

+ Corresponding author: mirian@ioc.fiocruz.br

(D) https://orcid.org/0000-0003-2223-3289

Received 27 March 2020

Accepted 22 December 2020 entry.(5) Different $T$. cruzi invasion mechanisms involving IP3-kinase activation, lysosome recruitment (including oligopeptidase B and cruzipain-mediated), sphingomyelinase-mediated membrane repair and autophagic pathways, as well as host cell cytoskeleton mechanisms, have been described..$^{(5)}$ Many cellular signaling pathways have been reported as driving parasite entrance, including protein kinase activation. ${ }^{(5)}$

The activation of focal adhesion kinase (FAK), a non-receptor tyrosine kinase, may be subverted by diverse intracellular pathogens, ${ }^{(6)}$ including $T$. cruzi. $^{(7)}$ FAK comprises three domains, a kinase domain flanked by two non-catalytic domains, the FERM (ezrin-radixin-moesin) domain at the N-terminus and the focal adhesion targeting (FAT) domain, a kinase domain regulator ${ }^{(8)}$ at the $\mathrm{C}$-terminus, which binds to focal adhesion proteins such as paxillin and talin, as well as docking sites for Src homology 3 (SH3) domain-containing proteins. ${ }^{(6)}$ FAK autophosphorylation at $\mathrm{Tyr}^{397}\left(\mathrm{pY}^{397}\right)$ recruits members of the Src family, amplifying FAK activation through the phosphorylation of additional residues (Y576, Y577, Y861 and Y925).(9)

Subversion of the FAK/Src signaling pathway modulates cardiomyocyte infection by $T$. cruzi. ${ }^{(7)}$ FAK activation, evidenced by FAK Tyr ${ }^{397}$ and c-Src phosphorylation, mediates parasite invasion. FAK inhibition by pharmacological inhibitors (PF573228), silencing by small interfering RNA (siRNA) or reduced expression by tetracycline-uninduced Tet-FAK(WT)-46 cells have 
been noted to impair $T$. cruzi entry into host cells, supporting FAK signaling involvement as a mechanism of T. cruzi invasion. ${ }^{(7)}$ However, the membrane surface receptor and/or ligands involved in this process have not yet been elucidated.

HSPG and integrin orchestrate FAK signaling pathway activation, ${ }^{(10,11)}$ and both are known to trigger $T$. cruzi-mammalian cell entry. ${ }^{(12,13)}$ Our previous data highlight HSPG, a cell-surface and extracellular matrix molecule composed of repeating hexuronic acid (D-glucuronic acid (GlcA) or L-iduronic acid (IdoA)) and D-glucosamine disaccharide units, as a T. cruzi invasion mediator in cardiomyocytes, the most affected cells during human infection. ${ }^{(14)}$ In addition, we have previously identified T. cruzi heparin binding proteins (HBP), presenting 65.8 and $59 \mathrm{kDa}$ molecular masses, that bind to heparin and heparan sulfate (HS). ${ }^{(15)}$ Thus, we hypothesise that $T$. cruzi-HSPG interaction triggers the FAK signaling pathway, leading to parasite invasion. In the present study, we evaluated the role of HSPG in triggering the FAK signaling pathway during the T. cruzi invasion process. Our results demonstrate that HSPG acts as a key molecule in FAK signaling pathway activation, regulating $T$. cruzi entry.

\section{MATERIALS AND METHODS}

Cell cultures - Primary cardiac muscle cell cultures were obtained as described previously. ${ }^{(16)}$ Cardiac ventricles obtained from 18-day-old mouse fetuses were fragmented and enzymatically dissociated with a tryp$\sin (0.025 \%)$ and collagenase $(0.01 \%)$ solution. Isolated cells were cultivated in Dulbecco's modified Eagle medium (DMEM) containing 7\% foetal bovine serum (FBS; Cultilab, São Paulo, Brazil), $2.5 \mathrm{mM} \mathrm{CaCl}, 1 \mathrm{mM}$ $L$-glutamine (Sigma), $2 \%$ chicken embryo extract and $1 \%$ penicillin/streptomycin solution (Life Technologies, São Paulo, Brazil) and maintained at $37^{\circ} \mathrm{C}$ under a $5 \%$ $\mathrm{CO}_{2}$ atmosphere. All animal procedures were approved by the IOC Committee of Ethics for the Use of Animals (CEUA license LW15-17).

Chinese hamster ovary (CHO) cell lines, comprising the wild type (CHO-K1) and a mutant type deficient in xylosyltransferase (CHO-745), and African green monkey kidney epithelial (Vero) cells were grown in a DMEM/F-12 nutrient mixture medium (GIBCO, Life Technologies, UK) and RPMI 1640 medium (Cultilab), respectively, supplemented with 5-10\% FBS, $1.176 \mathrm{~g} / \mathrm{L}$ sodium bicarbonate, $1 \mathrm{mM} L$-glutamine and antibiotics. At $80-100 \%$ confluence, cells were subcultured by dissociation with a trypsin-EDTA solution and maintained in a culture medium at $37^{\circ} \mathrm{C}$ in a humidified atmosphere containing $5 \% \mathrm{CO}_{2}$.

Parasites and T. cruzi-host cell interaction - Tissue culture-derived trypomastigotes (TCT) from two distinct T. cruzi lineages, Dm28c clone (TcI) and Y strain (TcII), were isolated from T. cruzi-infected Vero cells on the 4th day post-infection (dpi). Free trypomastigotes were harvested from the culture supernatant and used to infect the cardiomyocyte cultures and $\mathrm{CHO}$ lineages at a ratio of 20 parasites per host cell (20:1).
Cell treatment - Cardiomyocytes grown on $60 \mathrm{~mm}$ culture dishes $\left(2 \times 10^{6}\right.$ cells $)$ were treated for $24 \mathrm{~h}$ at $37^{\circ} \mathrm{C}$ with $2.5 \mathrm{mM} p$-nitrophenyl $\beta$-D-xylopyranoside ( $p$-n-xyloside) or $80 \mathrm{mU} / \mathrm{mL}$ heparinase I in serum-free DMEM. Two hours prior infection, the medium was substituted by a fresh medium containing $p$-n-xyloside or heparinase I. Additionally, CHO-k1 (6 x 10 $10^{5}$ cells) and CHO-745 (8 x $10^{5}$ cells) were seeded in $60 \mathrm{~mm}$ culture dishes in a DMEM/F-12 medium supplemented with $10 \%$ FBS. The cells were then infected with T. cruzi (Y strain or Dm28c clone) for $1 \mathrm{~h}$ at $37^{\circ} \mathrm{C}$. After interaction, free trypomastigotes were removed by washing with phosphate-buffered saline (PBS) and the cultures were processed for immunoblotting assay assessments. Controls consisted of the omission of $p$-n-xyloside and the applied enzyme. All experiments were performed at least three times.

Cell infection profile - Isolated cardiomyocytes (1 x $10^{5}$ cells) and $\mathrm{CHO}$ cells, CHO-k1 ( 1 x $10^{5}$ cells) and CHO-745 $\left(1.5 \times 10^{6}\right)$, were plated on 24 -well plates containing gelatin-coated glass coverslips. Heparinase I and $p$-n-xyloside-treated and untreated cardiomyocytes and CHO cells (CHO-k1 and CHO-745) were infected with T. cruzi (Y strain or Dm28c clone) for $1 \mathrm{~h}$ or $2 \mathrm{~h}$ at $37^{\circ} \mathrm{C}$. Additionally, CHO-k1 cells were treated for $1 \mathrm{~h}$ at $37^{\circ} \mathrm{C}$ with $20 \mu \mathrm{g} / \mathrm{mL}$ PF573228, a pharmacological FAK inhibitor, prior to T. cruzi interactions. After infection, the cells were fixed for $5 \mathrm{~min}$ with Bouin's solution, stained with Giemsa, dehydrated in an acetone-xylol series and mounted in Permount. The infection level was determined by the random quantification of at least 300 cells using a Zeiss Axioplan microscope. Experiments were carried out at least three times, in duplicate.

Indirect immunofluorescence - Uninfected and $T$. cruzi-infected cells, pretreated or not with $p$-n-xyloside or heparinase I, were fixed for $20 \mathrm{~min}$ at $4^{\circ} \mathrm{C}$ with $4 \%$ paraformaldehyde (Sigma Aldrich, São Paulo, Brazil) in PBS. Subsequently, the cells were washed with the blockage buffer [PBS with $4 \%$ bovine serum albumin (BSA)] and incubated overnight $\left(4^{\circ} \mathrm{C}\right)$ with the anti-FAK $\mathrm{Tyr}^{397}$ primary antibody (1:200, Invitrogen, Frederick, $\mathrm{MD})$. After washing, the cells were incubated with the anti-mouse IgG Alexa Fluor 555 secondary antibody (ThermoFisher). Actin filaments were visualised with AlexaFluor 488-labeled phalloidin (1:1000, ThermoFisher) and DNA was detected with 4,6-diamidino2-phenylindole dihydrochloride (DAPI). The analysis was performed using a Zeiss AxioImage M2 microscope with Apotome system.

Protein extraction and immunoblotting assay - Cell monolayers were washed with cold PBS (pH 7.4) and lysed with $50 \mathrm{mM}$ Tris- $\mathrm{HCl}$ containing $1 \%$ Triton X-100 and protease and phosphatase inhibitors. Total protein amounts were determined by the Follin-Lowry method. Protein extracts $(20 \mu \mathrm{g})$ were resolved by sodium dodecyl sulphate-polyacrylamide gel electrophoresis (SDS-PAGE) and transferred onto nitrocellulose membranes. The membranes were then blocked for 30-60 min in Tris-buffered saline containing $0.1 \%$ Tween 20 (TBST) and $5 \%$ bovine serum albumin (BSA) (TBST- 
BSA) before incubation with anti-phosphorylated FAK (1:1000; Invitrogen, Frederick, MD) or anti-FAK antibody (1:500; Santa Cruz Biotechnology). After washing with TBST, the membranes were incubated with horseradish peroxidase (HRP)-conjugated anti-rabbit IgG antibody (1:5000), revealed by chemiluminescence (ThermoFisher) and exposed to X-ray films. Densitometric analyses were performed using the ImageJ software. The anti-glyceraldehyde 3-phosphate dehydrogenase (GAPDH) antibody (Life Technologies) was used as the loading control. The immunoblotting experiments were performed independently three times.

Statistical analyses - The Student's $t$-test was used to determine the significance of differences between the mean values of three assays. A $p$ value $\leq 0.05$ was considered significant.

\section{RESULTS}

With the knowledge that HSPG orchestrates T. cruzi invasion and also regulates FAK signaling, which is, in turn, involved in T. cruzi entry into target cells, we questioned whether T. cruzi-HSPG interplay regulates FAK activation during the invasion process. The participation of HSPG as a T. cruzi entrance FAK activation regulator was investigated using two T. cruzi stocks, the Dm28c clone (TcI) and Y strain (TcII). Two different strategies were employed to determine the role of HSPG in this process, namely HS removal from the surface of cardiomyocytes with heparinase I and proteoglycan synthesis inhibition by $p$-n-xyloside, which abolishes the attachment of glycosaminoglycans to the core protein of proteoglycans by competing for endogenous xylose. As expected, $T$. cruzi-induced the FAK signaling pathway up-regulation in cardiomyocytes, leading to a significant increase in FAK Tyr ${ }^{397}$ phosphorylation levels after $1 \mathrm{~h}$, of $51 \%$ and $31 \%$ for the $\mathrm{Y}$ strain and Dm28c clone, respectively (Fig. 1). Cardiomyocyte treatment with $p$-n-xyloside or hepa- rinase I inhibited FAK signaling pathway activation by T. cruzi. HS removal from the surface of cardiomyocytes with heparinase I resulted in a 34\% FAK downregulation, whereas the $p$-n-xyloside treatment achieved a $28 \%$ reduction in FAK Tyr ${ }^{397}$ phosphorylation levels (Fig. 1) compared to untreated T. cruzi-infected cells. Such FAK autophosphorylation ( $\mathrm{Tyr}^{397}$ residue) inhibition was further accentuated in the interaction with Dm28c clone trypomastigotes, reaching maximum inhibition levels of $46 \%$ and $50 \%$ after cardiomyocyte treatment with $p$ $\mathrm{n}$-xyloside and heparinase I, respectively (Fig. 1). A reduction in FAK activation was observed only in T. cruzi Dm28c-infected cells compared to the phospho-FAK expression in uninfected cultures, suggesting that intrinsic $T$. cruzi genotype characteristics may differentially modulate FAK activation. However, a more detailed analysis is required to clarify this issue. Furthermore, FAK $\mathrm{Ty}^{397}$ distribution was also analysed by immunofluorescence staining. Intense FAK Tyr ${ }^{397}$ labeling was observed throughout the cell cytoplasm, as well as in focal adhesion sites of $T$. cruzi-infected cardiomyocytes compared to uninfected cells (Fig. 2). The fluorescence analyses were consistent with the immunoblotting data, indicating loss of FAK Tyr ${ }^{397}$ signal in infected cardiomyocytes treated with heparinase I or $p$-n-xyloside prior to $T$. cruzi infection (Fig. 2).

In addition, both $\mathrm{CHO}$ lineages were employed to investigate if the HSPG-T. cruzi interaction triggers the FAK signaling pathway. The lack of xylosyltransferase activity in CHO-745 cells, leading to glycosaminoglycans (GAG) deficiency, makes this model a suitable tool for HSPG-mediated signaling studies. Subsequently, we evaluated whether HSPG is a key downstream effector of the FAK signaling pathway during invasion by $T$. cruzi of both wild-type and mutant $\mathrm{CHO}$ cells. Upregulation of FAK phosphorylation was evidenced in wild-type $\mathrm{CHO}$ cells (CHO-K1) infected by T. cruzi. The infection by try-
A
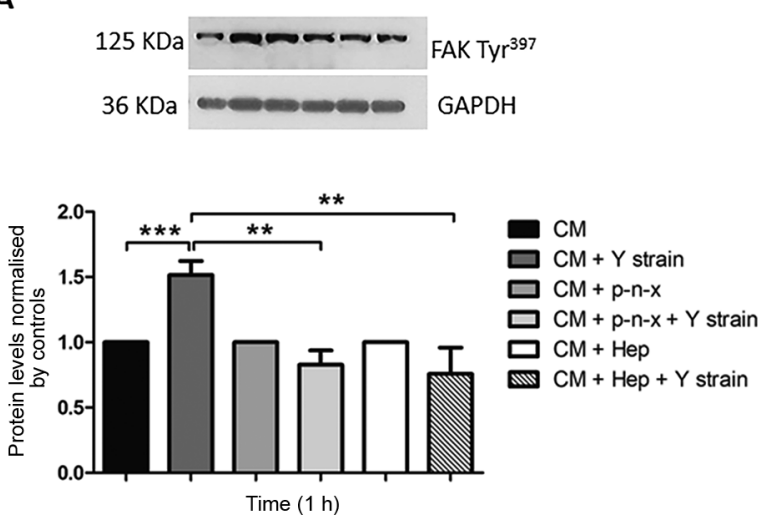

B
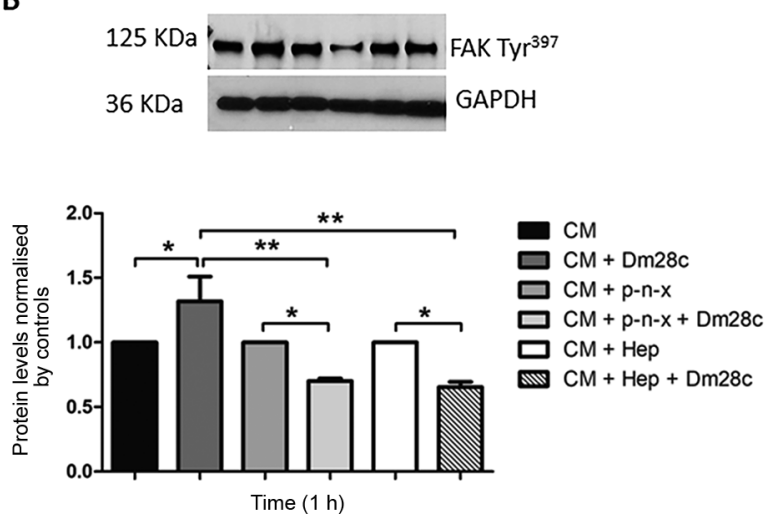

Fig. 1: heparan sulfate proteoglycans (HSPG) trigger focal adhesion kinase (FAK) activation during Trypanosoma cruzi-cardiomyocyte invasion. Glycosaminoglycans (GAGs) and heparan sulfate (HS) depletion after cardiomyocyte treatment with $2.5 \mathrm{mM}$-n-xyloside, and heparinase I $(80 \mathrm{mU})$ respectively, prior to Y strain T. cruzi infection, inhibits FAK Tyr ${ }^{397}$ up-regulation evidenced in untreated T. cruzi-infected cells (A). A western blotting analysis revealed significant increases, of $51 \%$ and $31 \%$, in FAK Tyr ${ }^{397}$ phosphorylation levels after Y strain (A) and Dm $28 \mathrm{c}$ clone (B) interactions with cardiomyocyte, respectively. FAK activation down-regulation, reaching $46 \%$ and $50 \%$ FAK Tyr ${ }^{397}$ level reductions, was achieved after cardiomyocyte treatment with heparinase I and $p$-n-xyloside, respectively, compared to untreated Dm $28 \mathrm{c}$ clone $T$. cruzi-infected cardiomyocytes (B). GAPDH was used as the housekeeping control. $\left(^{*}\right) \mathrm{p} \leq 0.05$ and $\left(^{* *}\right) \mathrm{p} \leq 0.004$, as determined by the Student's $t$-test. 


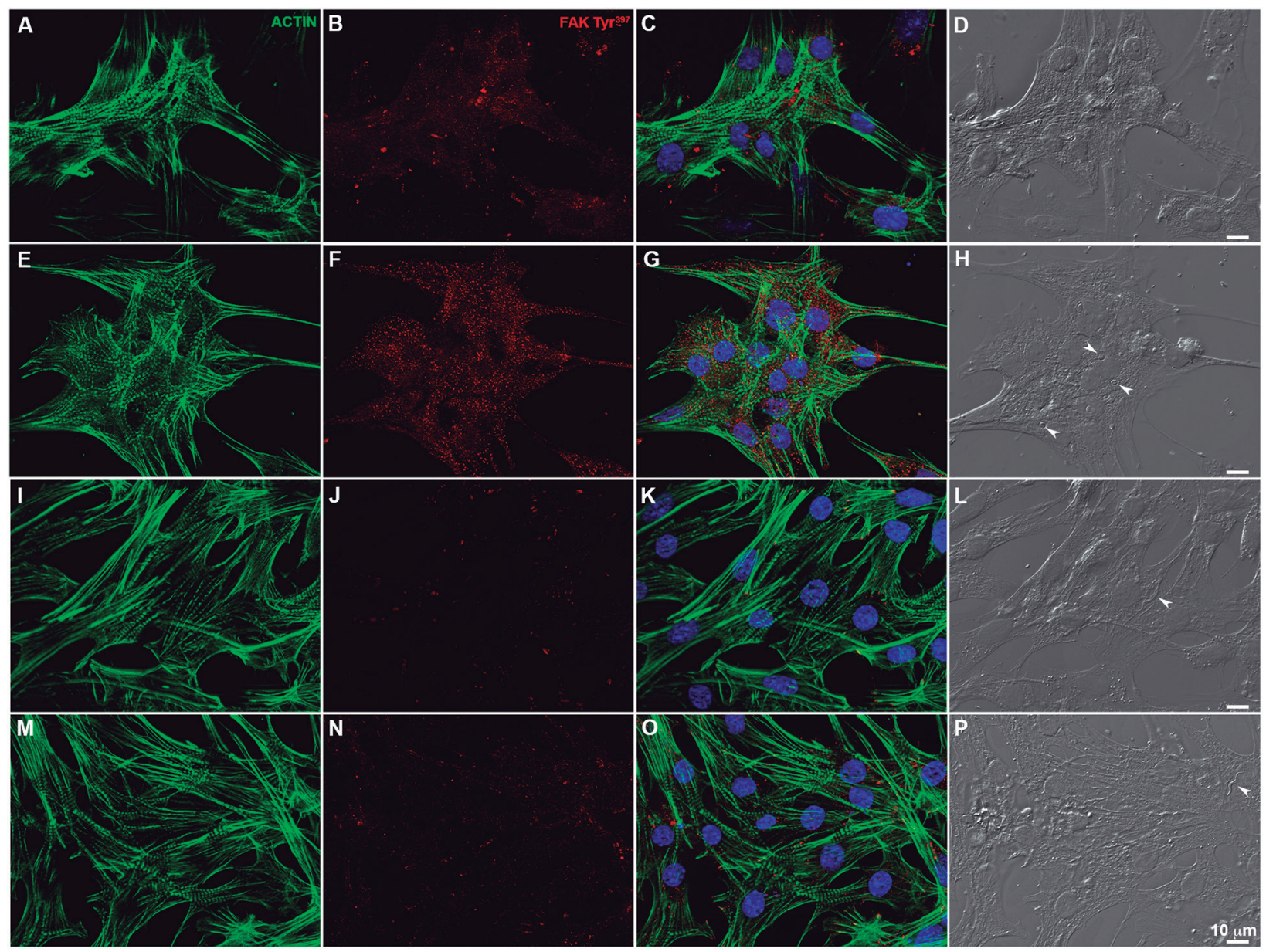

Fig. 2: heparan sulfate proteoglycans (HSPG) depletion in cardiomyocytes inhibits focal adhesion kinase (FAK) activation (FAK Tyr ${ }^{397}$ ) during Trypanosoma cruzi invasion. Actin filament (green) and FAK Tyr ${ }^{397}$ (red) distributions in uninfected (A-D) and T. cruzi-infected (E-P) cardiomyocytes. Well-developed myofibrils were visualised by phalloidin-Alexa 488 staining (green; A, E, I, M). An intense FFAK Tyr ${ }^{397}$ labeling (red) was noticed in cardiomyocytes after $T$. cruzi (Y strain) interaction ( $1 \mathrm{~h} ; \mathrm{F}$ ) compared to uninfected cells (B). Cardiomyocyte treatment with p-n-xyloside (I-L) or heparinase I (M-P) prior to T. cruzi infection (1 h) resulted in FAK activation inhibition, revealed by a weak FAK Y ${ }^{397}$ signal (red; J and N). DAPI, a DNA dye, stained the host cell nucleus and parasite nuclei and kinetoplasts (C, G, K, O). Intracellular parasites (arrowheads) are well-visualised by differential interference contrast (DIC; H, L, P). Merge images (C, G, K and O). Bars $=10 \mu \mathrm{m}$.

pomastigotes of both the Y strain and the Dm28c clone of CHO-K1 induced a 30\% increase in FAK phosphorylation (FAK Tyr ${ }^{397}$ ) levels (Fig. 3). In contrast, the infection of CHO 745, glycosaminoglycan-deficient cells, did not stimulate FAK activation. FAK phosphorylation $\left(\right.$ FAK $\mathrm{Ty}^{397}$ ) levels remained comparable to those of uninfected CHO-745 cells. Total FAK levels were unaltered in T. cruzi-infected CHO-k1 and CHO-745 cells (Fig. 3). Thereafter, we investigated whether FAK activation inhibition was correlated to GAG recognition and T. cruzi invasion, assessing both CHO-k1 and CHO-745 infection profiles (Fig. 4). As expected, GAG depletion, mainly HS, from the cell surface impaired parasite entry. Both T. cruzi stocks reached $36 \%$ of parasite invasion inhibition in GAG-deficient cells. Infection levels were $66 \%$ and $74 \%$ in wild type cells (CHO-k1) and $42 \%$ and $47 \%$ in mutant cells (CHO-745) for the Dm28c clone and Y strain, respectively (Fig. 4). As expected, treatment of CHO-k1 cells with the FAK pharmacological inhibitor (PF573228) also reduced parasite entry. Levels of 33\% and $29 \%$ infection, representing $49.5 \%$ and $60 \%$ inhibition, were observed for the T. cruzi Dm28c clone and Y strain, respectively (Fig. 4). T. cruzi invasion impairment is clearly observed in Fig. 4C, highlighting the expressive reduction of intracellular parasites in GAG-deficient cells (CHO-745) and PF573228 treated CHO-k1 cells.

\section{DISCUSSION}

Kinase signaling has been highlighted in the regulation of different biological events involved in target cell-pathogen interactions. ${ }^{(17,18)}$ Activation of signaling pathways during the initial parasite-host cell interface stage promotes $T$. cruzi entry by cytoskeleton-dependent or -independent processes. ${ }^{(5)}$ FAK, which is involved in cytoskeleton regulation, ${ }^{(19)}$ is activated during $T$. cruzi cardiomyocyte invasion. ${ }^{(7)}$ In the present study, we identified HSPG as protagonists in triggering the FAK signaling pathway during $T$. cruzi cell entry.

Parasite surface protein repertoires, capable of triggering different invasion mechanisms, are key to suc- 
A
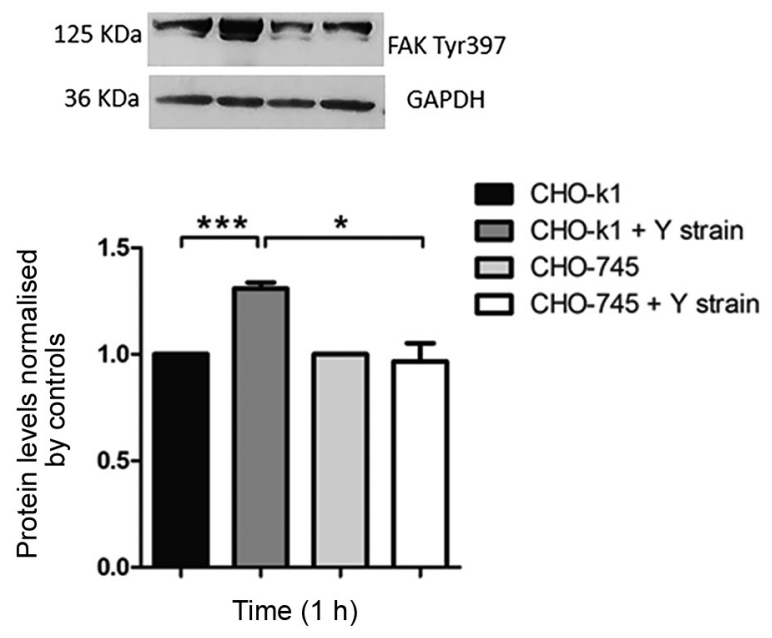

C
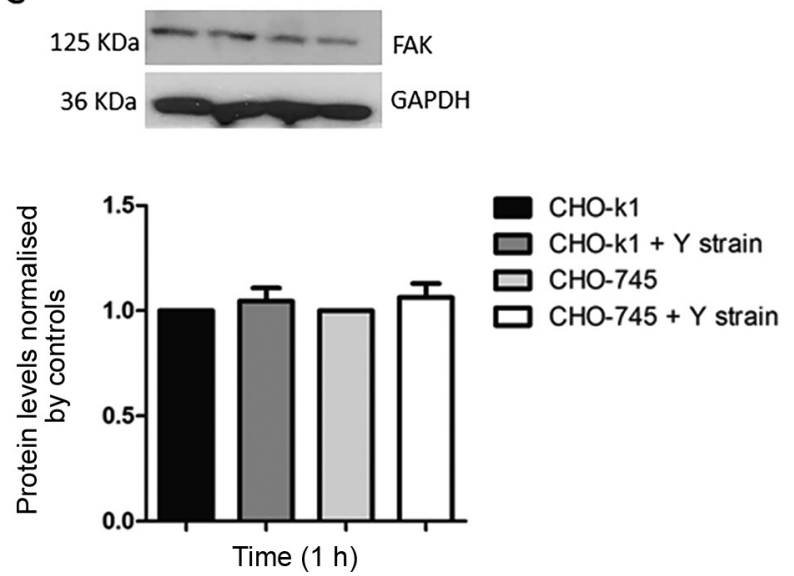

B
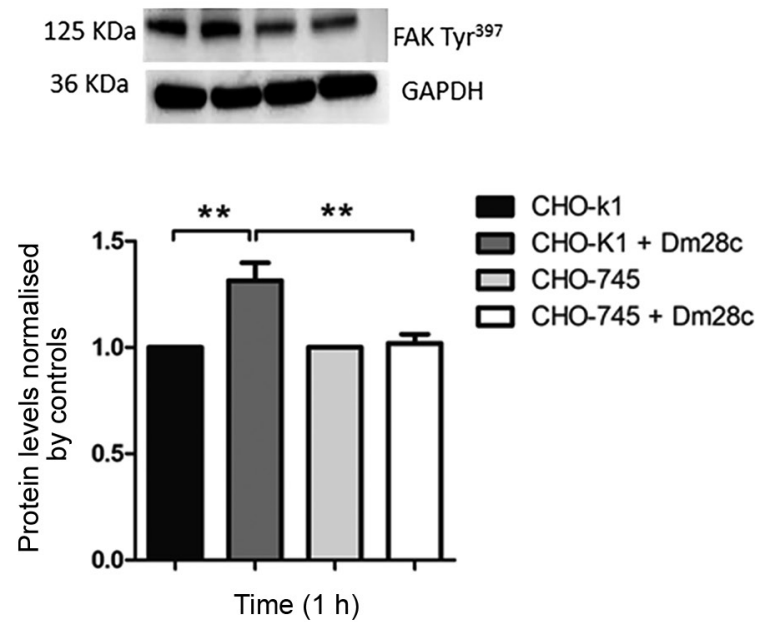

D
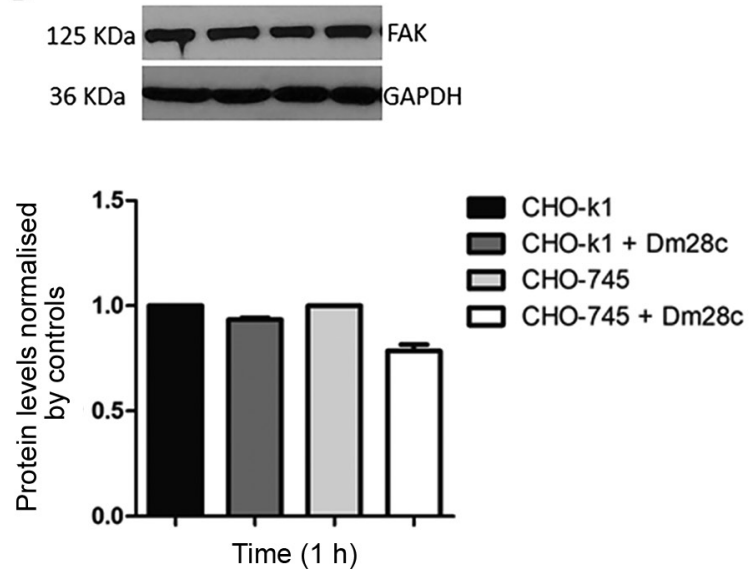

Fig. 3: focal adhesion kinase (FAK) signaling modulation in Chinese hamster ovary (CHO) cells invasion by Trypanosoma cruzi. Wild-type CHO cells (CHO-K1) and glycosaminoglycan (GAG)-deficient cells (CHO-745) were infected by the T. cruzi Y strain (A) and Dm28c clone (B), followed by immunoblotting FAK phosphorylation (FAK Tyr ${ }^{397}$ ) level detection. Both T. cruzi infections (Y strain and Dm28c clone) induced $30 \%$ increases in FAK phosphorylation (FAK Tyr ${ }^{397}$ ) levels in CHO-K1 cells (A and B). In contrast, T. cruzi-infected GAG-deficient cells (CHO745) did not elicit FAK signaling up-regulation (A and B). Total FAK levels were unaltered in CHO cells infected by both $T$. cruzi Y strain (C) and Dm28c clone (D). The anti-glyceraldehyde 3-phosphate dehydrogenase (GAPDH) signal was used to normalise loading differences between the lanes. $\left(^{*}\right) \mathrm{p} \leq 0.01$ and $(* *) \mathrm{p} \leq 0.005$, as determined by the Student's $t$-test.

cessful target cell entry. ${ }^{(20)}$ Our data demonstrates that $T$. cruzi strains belonging to different DTUs (TcI and TcII) modulate the FAK signaling pathway. Tissue-derived trypomastigotes (TCT) from the Y strain (TcII) and Dm28c clone (TcI) activate FAK signaling during cardiomyocyte invasion. Interestingly, it has been recently demonstrated that FAK activation is not required for metacyclic trypomastigote (MT) entry into host cells, ${ }^{(21)}$ showing that FAK depletion increases FAK-deficient HeLa cell susceptibility to MT invasion. ${ }^{(21)}$ This contrasting finding may be related to the differences between TCT and MT surface molecules involved in host cell invasion. In TCT, proteases, such as cruzipain and oligopeptidase B, as well as active and inactive trans-sialidases, play an important role in this process. ${ }^{(22)} \mathrm{Tc}-85$, a member of the gp85/ trans-sialidase superfamily, presents binding sites for laminin and cytokeratin 18 through the $\mathrm{NH} 2$ and $\mathrm{COOH}$ terminal domains, respectively, with the latter activating the ERK1/2 signaling pathway during parasite entry. An $85 \mathrm{kDa}$ protein, a TCT ligand that recognises the RGD binding motif in fibronectin, has also been implicated in the invasion process. Gp83 trans-sialidase, a ligand that binds a mammalian cell receptor (p74 receptor), modulates parasite entry by activating host tyrosine kinases in a PKC-dependent MAP kinase pathway. ${ }^{(23)}$ Integrin, a transmembrane receptor that regulates the extracellular matrix (ECM)-actin cytoskeleton crosstalk, also modulates $T$. cruzi entry. In addition, heparin-binding protein (HBP), a TCT surface molecule that binds heparin and $\mathrm{HS}$, promotes cell invasion in a proteoglycan-dependent manner. ${ }^{(15)}$ However, their MT surface expression has not yet been elucidated. Evidence has demonstrated that MT entrance relies on lysosome mobilisation, in a gp82- and gp90-dependent manner, ${ }^{(24)}$ but that lysosome biogenesis 
A

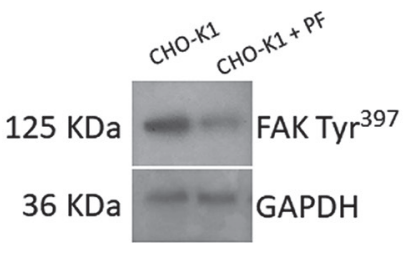

B

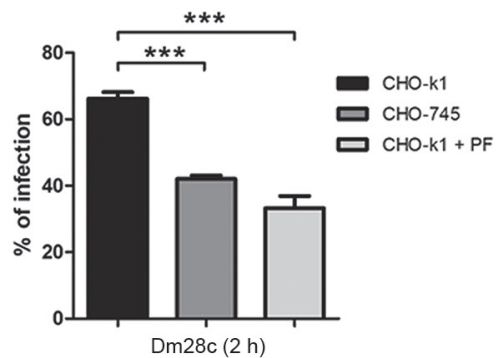

$\mathrm{CHO}-745$

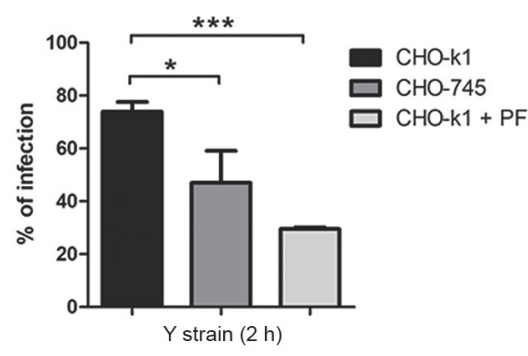

$\mathrm{CHO}-\mathrm{K} 1+\mathrm{PF}$
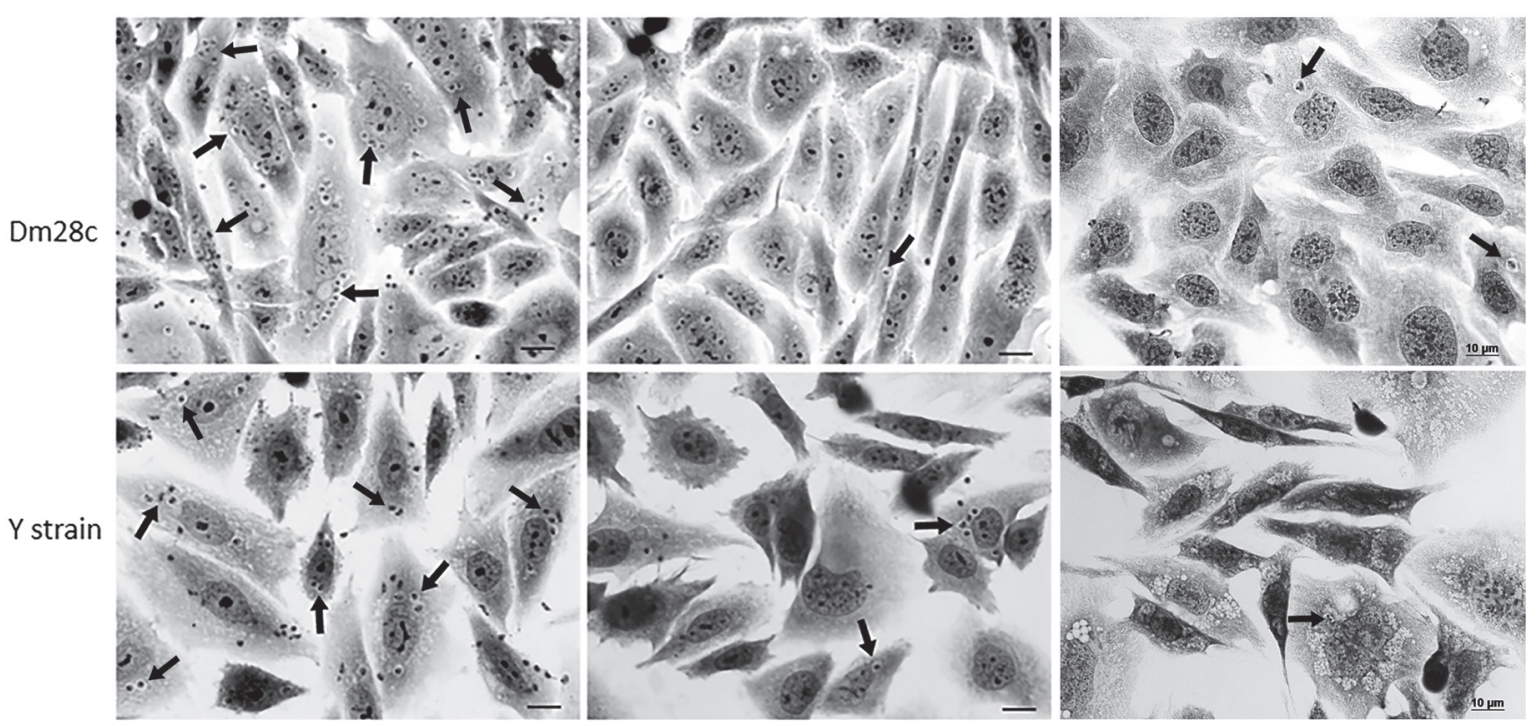

Fig. 4: glycosaminoglycans (GAGs) depletion from Chinese hamster ovary (CHO) cell surfaces impaired parasite entry. Wild type CHO-K1 cells displayed $66 \%$ and $74 \%$ of infection after interaction with Trypanosoma cruzi Dm28c clone (A) and Y strain (B), respectively, while infection levels were reduced to $42 \%$ and $47 \%$ in mutant cells (CHO-745). A 36\% inhibition of invasion was evidenced in GAG-deficient cells for both T. cruzi stocks. Additionally, treatment of CHO-K1 cells with PF573228 (PF), a pharmacological focal adhesion kinase (FAK) activation inhibitor, impaired parasite entry (B and C). Microscopy images show the T. cruzi infection profile in CHO cells. Reduced infection levels and intracellular parasites (arrows; C) are noteworthy in GAG deficient cells (CHO-745) and PF573228 treated CHO-k1 cells. $\left({ }^{*}\right) \mathrm{p} \leq 0.02$ and $(* * *)$ $\mathrm{p} \leq 0.0002$, as determined by the Student's $t$-test. Bar $=20 \mu \mathrm{m}$.

and spreading is not essential for TCT invasion. ${ }^{(25)} \mathrm{Ad}-$ ditionally, the signaling events triggered by gp35/50, a surface glycoprotein also expressed in MT, modulate cytoskeleton-dependent internalisation, ${ }^{(24)}$ demonstrating that the glycoprotein profile determines the invasion mechanism process.

FAK activation is known to be modulated by integrin and HSPG on mammalian cell surfaces. ${ }^{(26,27)} \mathrm{Re}-$ garding the fact that HSPG mediates $T$. cruzi host cell invasion, ${ }^{(13,14,15)}$ which seems to be modulated by HBP on the T. cruzi surface, HSPG participation as a receptor molecule resulting in downstream FAK activation was also evaluated. As expected, TCT activates the FAK signaling pathway in cardiomyocytes during the invasion process, revealed by FAK phosphorylation. This kinase activation, however, was inhibited, leading to increased resistance to TCT internalisation when HSPG was removed by heparinase I treatment or proteoglycan biosynthesis inhibition by $p$-n-xyloside, demonstrating HSPG involvement in eliciting FAK activation. The lack of a FAK phosphorylation fluorescence signal was also evidenced after GAG and HS removal, supporting the indication that the absence of HSPG down-regulates FAK autophosphorylation (FAK $\mathrm{Tyr}^{397}$ ), decreasing $T$. cruzi uptake by cardiac myocytes. FAK phosphorylation inhibition in GAG-deficient CHO cells, leading to TCT internalisation resistance, also corroborates the fact that HSPG is implicated in FAK activation during T. cruzi entry. However, we cannot disregard the integrin role during this process, whose participation in FAK activation has been suggested during $T$. cruzi-cardiac cell interactions ${ }^{(28)}$ but requires further investigation.

Modulation of the FAK signaling pathway by HSPG interactions has also been reported during Human 
Papillomavirus Type 16 (HPV16) invasion. ${ }^{(29)}$ HPV16 binding to HSPG to human transformed keratinocyte ( $\mathrm{HaCaT}$ cells) surfaces mediates interactions with $\alpha 6$ integrin, regulating FAK activation and the entry of viral particles by filopodia formation. Kaposi's sarcoma associated herpesvirus (KSHV) binding to human acute monocyticleukemia cell line (THP-1) also involves HS recognition, leading to increased FAK levels, extracellular signal-regulated kinase (ERK1/2) and phosphatidylinositol 3-kinase (IP3K) phosphorylation. ${ }^{(30)}$ Interestingly, HSPG participation in triggering FAK activation has also been evidenced in non-microbial events. Biological cellular processes in tissue physiology have been demonstrated as regulated by an interstitial flow in a 3-dimensional (3D) microenvironment. ${ }^{(31)}$ In this scenario, HSPG acts as a mechanosensory molecule, either integrin-dependent or independent, modulating cellular responses to the interstitial flow, leading to FAK activation and downstream ERK signaling, which regulates vascular cell motility.

Together, our data add new insights for a better understanding of FAK activation-mediated T. cruzi invasion. T. cruzi-HSPG recognition activates the FAK signaling pathway, mediating TCT entry into cardiomyocytes. Since activated FAK regulates members of the Rho GTPase family, including Rac, Cdc42 and RhoA, modulating actin cytoskeleton dynamics, it will be interesting to investigate the complex signaling network involved in this process in detail. A complete understanding of FAK-mediated parasite entry will shed light on T. cruzi cardiac cell invasion mechanisms.

\section{ACKNOWLEDGEMENTS}

To Mr Alanderson Nogueira for technical support and for the operation of the Apotome system.

\section{AUTHORS' CONTRIBUTION}

TGM and MCSP conceived and designed the proposal; TGM, EAC and MCSP performed lab experiments and processed the data; TGM and MCSP draft the manuscript; MCSP coordinated the resources. The authors declare no competing interest.

\section{REFERENCES}

1. Antinori S, Galimberti L, Bianco R, Grande R, Galli M, Corbellino M. Chagas disease in Europe: a review for the internist in the globalized world. Eur J Intern Med. 2017; 43: 6-15.

2. Rassi Jr A, Marin-Neto JA, Rassi A. Chronic Chagas cardiomyopathy: a review of the main pathogenic mechanisms and the efficacy of aetiological treatment following the BENznidazole Evaluation for Interrupting Trypanosomiasis (BENEFIT) trial. Mem Inst Oswaldo Cruz. 2017; 112(3): 224-35.

3. Lee BY, Bacon KM, Bottazzi ME, Hotez PJ. Global economic burden of Chagas disease: a computational simulation model. Lancet Infect Dis. 2013; 13(4): 342-8.

4. Nagajyothi F, Machado FS, Burleigh BA, Jelicks LA, Scherer PE, Mukherjee S, et al. Mechanisms of Trypanosoma cruzi persistence in Chagas disease. Cell Microbiol. 2012; 14(5): 634-43.

5. Barria E, Reignault LC, De Souza W. How does the main infective stage of Trypanosoma cruzi enter and avoid degradation in host cells? A description of the pathways and organelles involved on these processes. IntechOpen. 2019; DOI: http://dx.doi. org/10.5772/intechopen.86046.
6. Krishnan HH, Sharma-Walia N, Streblow DN, Naranatt PP, Chandran B. Focal adhesion kinase is critical for entry of Kaposi's sarcoma-associated herpesvirus into target cells. J Virol. 2006; 80(3): 1167-80.

7. Melo TG, Tucci AR, Nogueira AR, Meirelles MN, Pereira MC. The involvement of FAK and Src in the invasion of cardiomyocytes by Trypanosoma cruzi. Exp Parasitol. 2014; 139: 49-57.

8. Cooper LA, Shen TL, Guan JL. Regulation of focal adhesion kinase by its amino-terminal domain through an autoinhibitory interaction. Mol Cell Biol. 2003; 23(22): 8030-41.

9. Calalb MB, Polte TR, Hanks SK. Tyrosine phosphorylation of focal adhesion kinase at sites in the catalytic domain regulates kinase activity: a role for Src family kinases. Mol Cell Biol. 1995; 15(2): 954-63.

10. Bass MD, Humphries MJ. Cytoplasmic interactions of syndecan-4 orchestrate adhesion receptor and growth factor receptor signaling. Biochem J. 2002; 368(Pt 1): 1-15.

11. Hall JE, Fu W, Schaller MD. Focal adhesion kinase: exploring Fak structure to gain insight into function. Int Rev Cell Mol Biol. 2011; 288: $185-225$.

12. Fernandez MA, Munoz-Fernandez MA, Fresno M. Involvement of beta 1 integrins in the binding and entry of Trypanosoma cruzi into human macrophages. Eur J Immunol. 1993; 23(2): 552-7.

13. Ortega-Barria E, Pereira ME. A novel T. cruzi heparin-binding protein promotes fibroblast adhesion and penetration of engineered bacteria and trypanosomes into mammalian cells. J Eukaryot Microbiol. 1991; 50(2): 97-103.

14. Calvet CM, Toma L, De Souza FR, Meirelles MN, Pereira MC. Heparan sulfate proteoglycans mediate the invasion of cardiomyocytes by Trypanosoma cruzi. Parasitology. 2011; 138(5): 593-601.

15. Oliveira FO, Alves CR, Calvet CM, Toma L, Bouças RI, Nader $\mathrm{HB}$, et al. Trypanosoma cruzi heparin-binding proteins and the nature of the host cell heparan sulfate-binding domain. Microb Pathog. 2008; 44(4): 329-38.

16. Meirelles MN, de Araujo-Jorge TC, Miranda CF, de Souza W, Barbosa HS. Interaction of Trypanosoma cruzi with heart muscle cells: ultrastructural and cytochemical analysis of endocytic vacuole formation and effect upon myogenesis in vitro. Eur J Cell Biol. 1986; 41(2): 198-206.

17. Alto NM, Orth K. Subversion of cell signaling by pathogens. Cold Spring Harb Perspect Biol. 2012; 4(9): e006114.

18. Soni R, Sharma D, Rai P, Sharma B, Bhatt TK. Signaling strategies of malaria parasite for its survival, proliferation, and infection during erythrocytic stage. Front Immunol. 2017; 28: 349.

19. Hohmann T, Feese K, Ghadban C, Dehghani F, Grabiec U. On the influence of cannabinoids on cell morphology and motility of glioblastoma cells. PLoS One. 2019; 14(2): e0212037.

20. Osorio L, Ríos I, Gutiérrez B, González J. Virulence factors of Trypanosoma cruzi: who is who? Microbes Infect. 2012; 14(5): 1390-402.

21. Onofre TS, Rodrigues JPF, Yoshida N. Depletion of host cell focal adhesion kinase increases the susceptibility to invasion by Trypanosoma cruzi metacyclic forms. Front Cell Infect Microbiol. 2019; 26: 231.

22. Maeda FY, Cortez C, Yoshida N. Cell signaling during Trypanosoma cruzi invasion. Front Immunol. 2012; 3: 361.

23. Pius N, Lima MF, Johnson CA, Pratap S, Villalta F. Regulation and use of the extracellular matrix by Trypanosoma cruzi during early infection. Front Immunol. 2012; 6: 337.

24. Clemente TM, Cortez C, Novaes AS, Yoshida N. Surface molecules released by Trypanosoma cruzi metacyclic forms downregulate host cell invasion. PLoS Negl Trop Dis. 2016; 10(8): e0004883. 
25. Cortez C, Real F, Yoshida N. Lysosome biogenesis/scattering increases host cell susceptibility to invasion by Trypanosoma cruzi metacyclic forms and resistance to tissue culture trypomastigotes. Cell Microbiol. 2016; 18: 748-60.

26. Dreyfuss JL, Regatieri CV, Jarrouge TR, Cavalheiro RP, Sampaio LO, Nader HB. Heparan sulfate proteoglycans: structure, protein interactions and cell signaling. An Acad Bras Cienc. 2009; 81(3): 409-29.

27. Wilcox-Adelman SA, Denhez F, Goetinck PF. Syndecan-4 modulates focal adhesion kinase phosphorylation. J Biol Chem. 2002; 277(36): 32970-7.

28. Melo TG, Adesse D, Meirelles MN, Pereira MCS. Trypanosoma cruzi down-regulates mechanosensitive proteins in cardiomyocytes. Mem Inst Oswaldo Cruz. 2019; 114: 1-9.

29. Abban CY, Meneses PI. Usage of heparan sulfate, integrins, and FAK in HPV16 infection. Virology. 2010; 403(1): 1-16.

30. Kerur N, Veettil MV, Sharma-Walia N, Sadagopan S, Bottero V, Paul AG. Characterization of entry and infection of monocytic THP-1 cells by Kaposi's sarcoma associated herpesvirus (KSHV): role of heparan sulfate, DC-SIGN, integrins and signaling. Virology. 2010; 406(1): 103-16.

31. Shi ZD, Wang H, Tarbell JM. Heparan sulfate proteoglycans mediate interstitial flow mechanotransduction regulating MMP-13 expression and cell motility via FAK-ERK in 3D collagen. PLoS One. 2011; 6: e15956. 